OPEN ACCESS

Edited by:

Thomas Zilli,

Université de Genève,

Switzerland

Reviewed by:

Angel Montero, HM Madrid Hospital,

Spain

Tomoyuki Makino, Kanazawa University,

Japan

*Correspondence:

Mattia Zaffaron

mattia.zaffaroni@ieo.it

${ }^{\dagger}$ These authors share first authorship

Specialty section:

This article was submitted to Genitourinary Oncology, a section of the journal Frontiers in Oncology

Received: 29 June 2021

Accepted: 13 July 2021

Published: 04 August 2021

Citation:

Marvaso G, Corrao G, Zaffaroni M, Pepa M, Augugliaro M, Volpe $S$, Musi G, Luzzago S, Mistretta FA, Verri E, Cossu Rocca M, Ferro M,

Petralia G, Nolè F, De Cobelli O, Orecchia $R$ and Jereczek-Fossa BA (2021) Therapeutic Sequences in the

Treatment of High-Risk Prostate Cancer: Paving the Way Towards Multimodal Tailored Approaches.

Front. Oncol. 11:732766. doi: 10.3389/fonc.2021.732766

\section{Therapeutic Sequences in the Treatment of High-Risk Prostate Cancer: Paving the Way Towards Multimodal Tailored Approaches}

\author{
Giulia Marvaso ${ }^{1,2 \dagger}$, Giulia Corrao ${ }^{1,2 \dagger}$, Mattia Zaffaroni ${ }^{1 *}$, Matteo Pepa ${ }^{1}$, \\ Matteo Augugliaro ${ }^{1}$, Stefania Volpe ${ }^{1,2}$, Gennaro Musi ${ }^{2,3}$, Stefano Luzzago ${ }^{3}$, \\ Francesco Alessandro Mistretta ${ }^{3}$, Elena Verri ${ }^{4}$, Maria Cossu Rocca ${ }^{4}$, Matteo Ferro ${ }^{3}$, \\ Giuseppe Petralia $^{2,5}$, Franco Nolè ${ }^{6}$, Ottavio De Cobelli ${ }^{2,3}$, Roberto Orecchia $^{7}$ \\ and Barbara Alicja Jereczek-Fossa ${ }^{1,2}$ \\ ${ }^{1}$ Division of Radiation Oncology, IEO, European Institute of Oncology IRCCS, Milan, Italy, ${ }^{2}$ Department of Oncology and \\ Hemato-Oncology, University of Milan, Milan, Italy, ${ }^{3}$ Department of Urology, IEO, European Institute of Oncology IRCCS, \\ Milan, Italy, ${ }^{4}$ Department of Medical Oncology, IEO, European Institute of Oncology IRCCS, Milan, Italy, 5 Precision Imaging \\ and Research Unit, Department of Medical Imaging and Radiation Sciences, IEO, European Institute of Oncology IRCCS, \\ Milan, Italy, ${ }^{6}$ Medical Oncology Division of Urogenital \& Head \& Neck Tumors, IEO, European Institute of Oncology IRCCS, \\ Milan, Italy, ${ }^{7}$ Scientific Directorate, IEO, European Institute of Oncology IRCCS, Milan, Italy
}

Various definitions are currently in use to describe high-risk prostate cancer. This variety in definitions is important for patient counseling, since predicted outcomes depend on which classification is applied to identify patient's prostate cancer risk category. Historically, strategies for the treatment of localized high-risk prostate cancer comprise local approaches such as surgery and radiotherapy, as well as systemic approaches such as hormonal therapy. Nevertheless, since high-risk prostate cancer patients remain the group with higher-risk of treatment failure and mortality rates, nowadays, novel treatment strategies, comprising hypofractionated-radiotherapy, second-generation antiandrogens, and hadrontherapy, are being explored in order to improve their long-term oncological outcomes. This narrative review aims to report the current management of high-risk prostate cancer and to explore the future perspectives in this clinical setting.

Keywords: high risk prostate cancer, personalized medicine, hadrontherapy, narrative review, second-generation antiandrogens, iPARP treatment

\footnotetext{
Abbreviations: ADT, Androgen Deprivation Therapy; AR, Androgen Receptor; CIRT, Carbon Ion Radiotherapy; CSS, Cancer Specific Survival; DDR, DNA Damage Repair; EAU, European Association of Urology; EANM, European Association of Nuclear Medicine; EBRT, External Beam Radiotherapy; ePLND, Extended Pelvic Lymph Nodes Dissection; ESTRO, European Society for Therapeutic Radiation and Oncology; ESUR, European Society of Urogenital Radiology; G, Grade; GI, Gastrointestinal; GU, Genitourinary; GS, Gleason Score; HR PCa, High-risk Prostate Cancer; iPARP, Enzyme Poly ADP Ribose Polymerase inhibitors; IMRT, Intensity Modulated Radiotherapy; LCR, Local Control Rate; mCRPC, metastatic Castration-Resistant Prostate Cancer; NIRS, National Institute of Radiological Sciences; NCCN, National Comprehensive Cancer Network; OARs, Organ at Risk; OS, Overall Survival; PBRT, Proton Beam Radiotherapy; PCSM, Prostate Cancer Specific Mortality; PSA, Prostate Specific Antigen; PTV, Planning Target Volume; QoL, Quality of Life; RBE, Relative Biological Effectiveness; RCT(s), Randomized Clinical Trial(s); RP, Radical Prostatectomy; SBRT, Stereotactic Body Radiation Therapy; SIOG, International Society of Geriatric Oncology; TCGA, The Cancer Genome Atlas; WPRT, Whole Pelvis Radiotherapy.
} 


\section{INTRODUCTION}

High-risk prostate cancer (HR PCa) is defined according to the pathological grade of the disease (Gleason score (GS)), prostate specific antigen (PSA) value, and disease extent (1). A summary of the currently available classifications is reported in Figure $\mathbf{1}$. The heterogeneity of patients included in this risk class accounts for the variety of expected outcomes with widely reported percentages of biochemical and metastatic recurrences (2-4). This non-homogeneity in definitions is important for patient counseling, as reported outcomes depend on which classification is applied to identify patient PCa risk category (5). Consequently, the ideal management strategy is presently unclear but is likely to involve a multimodal approach. Given that HR PCa is associated with early treatment failure and metastatic relapse of disease after definitive therapies, with low overall survival (OS) rates (6), novel treatment strategies are being explored in order to improve their long-term oncological outcomes.

This narrative review aims to investigate the evolution of the management of HR PCa. In particular, the first section reports some of the old, and partially solved, questions: (i) radiotherapy (RT) vs surgery, (ii) appropriate androgen deprivation therapy (ADT) duration, and (iii) prophylactic pelvic irradiation. The subsequent section focuses on future perspectives and novel treatment strategies including (i) ultra-hypofractionated RT, (ii) second-generation antiandrogens and poly (ADP-ribose) polymerase inhibitors (iPARP), and (iii) particle therapy.

\section{OLD SCENARIOS}

\section{Local Approaches: RT vs Surgery}

It is well established that treatment options for localized HR PCa should include a definitive local strategy, with 87 and 57\% cancer-specific survival (CSS) rates observed among treated and untreated patients, respectively (7). In accordance to these data, both National Comprehensive Cancer Network (NCCN) (8) and European Association of Urology (EAU) guidelines (1) strongly recommend a definitive treatment, stratifying patients in accordance with their life expectancy (with a threshold of 5 and 10 years, respectively). Guidelines' recommendations include radical prostatectomy $(\mathrm{RP})+$ pelvic lymph node dissection (PLDN) or external beam RT (EBRT) + long-term ADT (1.5-3 years) \pm a brachytherapy boost (8).

Since evidence from randomized controlled trials (RCTs) comparing surgery and EBRT still lacks, nowadays no consensus exists on the best treatment choice. A recent

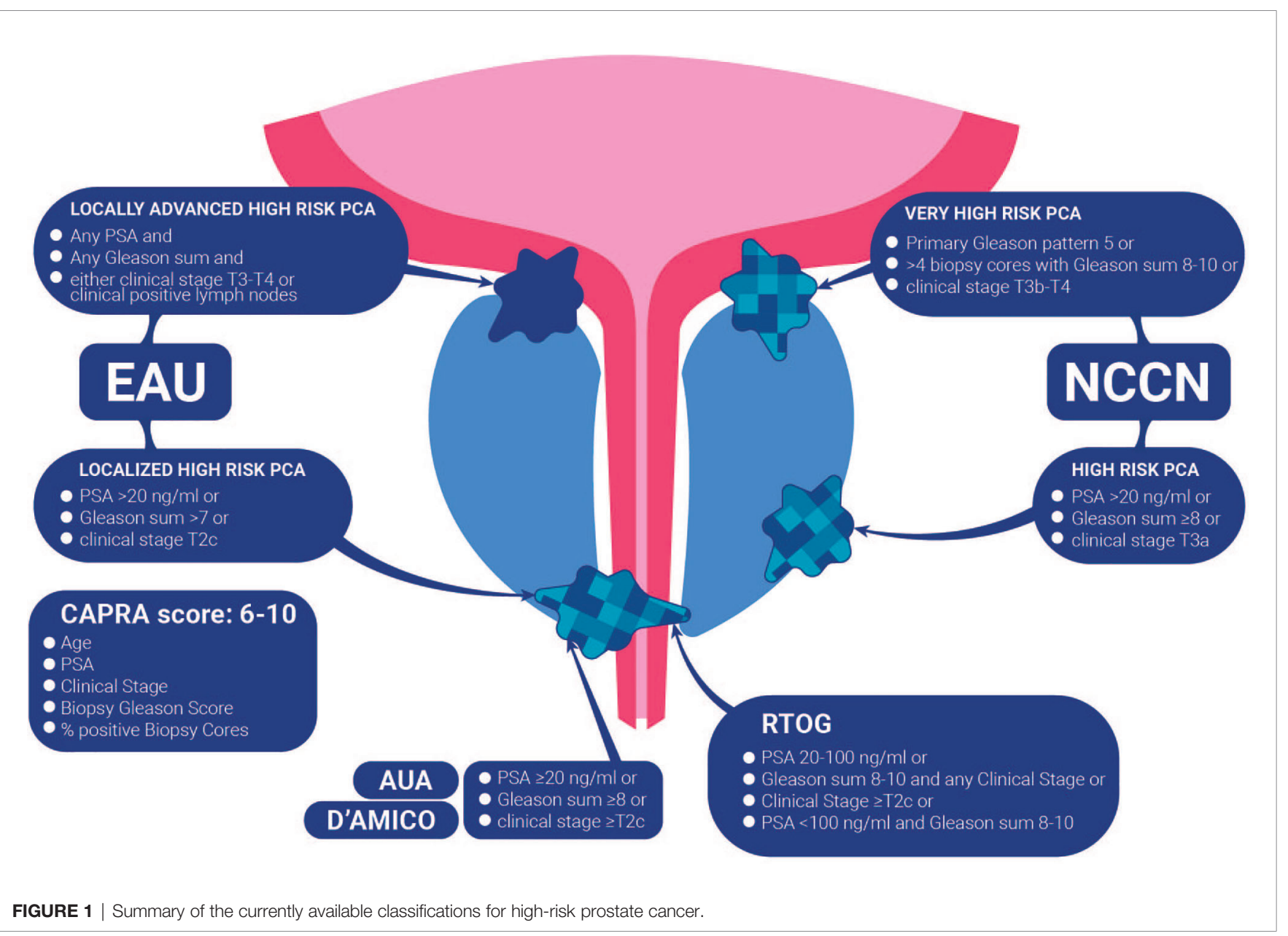


international multidisciplinary systematic review (9) was unable to demonstrate the superiority of such approaches as primary local therapy. The ongoing randomized phase III SPCG-15 trial (10) comparing CSS of locally advanced PCa patients treated with $\mathrm{RP}+\mathrm{ePLDN} \pm \mathrm{EBRT}$ or $\mathrm{EBRT}+\mathrm{ADT}$ is expected to provide evidence on this aspect.

\section{EBRT + ADT}

Androgen suppression is an established strategy for the treatment of HR PCa. Usually it is accomplished via the use of luteinizing hormone-releasing hormone (LHRH) analogs or antagonists, \pm antiandrogens.

It is widely recognized that improving OS may be obtained by adding ADT to RT in HR PCa patients with a life expectancy $>10$ years (11-13). The latter evidence is supported by an RCT showing 10 -year OS of 40 to $58 \%$ among patients receiving RT alone or combined treatments, respectively ( $p=0.0004)$.

However, the appropriate ADT duration is actually undefined, considering in particular its relation with the patient's reported quality of life (QoL). Two studies $(14,15)$ addressing this issue have reported that long-term ADT (18-36 months) has better oncological outcomes with respect to shortterm ADT. Conversely, a recent phase III RCT (16) comparing long- (36 months) and intermediate- (18 months) term ADT did not observe significant difference in clinical outcomes (CSS and distant metastases development), but only a benefit in QoL for intermediate group. Currently, age, performance status, comorbidities, and the number of poor prognostic factors are recommended to be considered for establishing the ADT duration in clinical practice. In general, the current evidence supports the fact that any ADT duration is better than no ADT at all (12, 17-19), that long-term ADT (e.g., 3 years) is slightly better in OS than a short duration (6 months) (15), but it remains debated whether a duration of $<3$ years (16) in some patients or $>3$ years in very HR patients is more appropriate.

\section{Whole-Pelvis Irradiation}

The efficacy of dose-escalated EBRT to the prostate alone in patients with HR disease might be limited by the increased likelihood of occult pelvic lymph node metastases outside of the radiation field.

In the past years, different studies showed opposing evidences on the administration of prophylactic whole-pelvis RT, such as the study by Roach et al. (20), which reported a benefit for neoadjuvant ADT followed by WPRT in contrast with Lawton and Colleagues (21). who failed to demonstrate a benefit in men without positive lymph nodes.

As a result, there is no consensus on WPRT administration. Ideally, WPRT should erase micrometastatic lesions, improving locoregional control (LRC) and OS.

However, the phase III POP-RT trial (22), including 224 patients affected by HR-PCa receiving hypofractionated RT to the prostate and randomized to include or exclude the whole pelvis, confirms the role of prophylactic WPRT in improving the biochemical failure-free survival and disease-free survival without a significant reported benefit in OS rates.
For this purpose, two RCTs $(23,24)$ are ongoing and might provide more robust evidence on the issue. In particular, with an expected trial end date of August 2021, PIVOTAL-boost is a multicenter four-arm superiority phase III trial for intermediate and HR PCa patients with failure-free survival as primary endpoint through administration of intensity-modulated RT (IMRT) on prostate \pm pelvic and prostate boost on dominant lesion(s). Similarly, the RTOG 0924, a phase III randomized trial, with primary outcome measure stated as OS assigning unfavorable intermediate or favorable $\mathrm{HR}$ PCa patients to $\mathrm{ADT}+\mathrm{EBRT} \pm \mathrm{WPRT}$. The estimated primary completion date is July 2027.

Waiting for results from these RCTs, radiation oncologists are divided on the best strategy in the clinical practice.

In the era of tailored treatments, in order to avoid unnecessarily larger treatment fields, Gallium 68 prostate specific membrane antigen ( $\mathrm{Ga}^{68}$ PSMA-PET) and whole-body Magnetic Resonance Imaging (MRI) could help to early identify pelvic lymph node localizations if PSA is still detectable $(25,26)$.

Such image-guidance techniques, mapping microscopic disease with improved sensitivity and sensibility, could also allow for dose escalation to nodes outside the conventional volumes (27).

\section{NEW SCENARIOS}

\section{Hypofractionated and Ultra- Hypofractionated RT}

Based on the radiosensibility of the PCa cells, it has been largely demonstrated that hypofractionation and extreme hypofractionation are safe and effective in low and intermediate risk PCa (28-31). In fact, the strong biologic rationale behind hypofractionation is based on the theory that the slow proliferation of $\mathrm{PCa}$ cells results in a different radiation response compared to other human cancers $(32,33)$. Therefore, the inability of PCa cells to overcome the higher rate of DNA damage induced by each fraction translates into an increased sensitivity to higher doses per fraction.

Up to date, multiple clinical trials have shown the effectiveness and the safety of moderate/standard hypofractionation for PCa treatment both in terms of oncological outcomes and toxicity (28-30, 34-36). Currently, thanks to the advent of modern techniques such as IMRT, highly conformal doses can be delivered to the target without affecting normal tissues, tilting the risk/benefit ratio more favorably towards RT $(37,38)$. Based on results from CHHiP and HYPRO (30) trials, hypofractionated schemes represent a valid treatment option for HR PCa. The number of studies involving extreme hypofractionation (defined as the delivery of $5-10 \mathrm{~Gy} /$ fraction in four to seven fractions) is relatively low, and a direct comparison of different hypofractionation schemes is still lacking. Therefore, despite being cited in clinical practice guidelines next to moderate hypofractionation schemes, the current level of evidence is too low to implement extreme hypofractionation as a standard of care (39). 
Overall, while hypofractionation is a well-established practice, the comparison and reproducibility of published studies regarding ultra-hypofractionation in $\mathrm{HR}$ PCa are of difficult interpretation due to the many limitations that must be taken into account in order to draw reliable conclusions including (i) the small number of prospective studies, (ii) the paucity and quality of the reported data, (iii) the lack of technical RT delivery data analyses, (iv) the scarcity of QoL data, and (v) the not consistent definition of HR PCa throughout the studies.

To explore the feasibility of ultra-hypofractionated regimens, the Hypo-RT trial (40) enrolled 1,200 intermediate and HR PCa with about $10 \%$ being HR patients who randomly received ultrahypofractionated (42.7 Gy in seven fractions) or conventional RT (78 Gy in 39 fractions). Results at 5 years reported a failure-free survival rate of $84 \%$ in both arms (no ADT was allowed). Posthoc subgroup analyses failed to show significant interactions between risk and treatment group.

One hundred intermediate or HR PCa patients enrolled in the hypo-FLAME (41) prospective phase II trial received 35 Gy in five weekly fractions to the whole prostate gland with an integrated boost up to $50 \mathrm{~Gy}$ to the dominant intraprostatic lesion(s). In the study, no grade (G) 3 acute genitourinary (GU) or gastrointestinal (GI) toxicities were observed.

One of the main issues about ultra-hypofractionated regimens in HR PCa regards the expected toxicity following prophylactic WPRT.

In this scenario, the FASTR and FASTR-2 trials $(42,43)$ aimed to evaluate acute toxicity after ultra-hypofractionated RT.

In the first FASTR study, 15 men matched the inclusion criteria. RT was delivered to the prostate gland (40 Gy) and simultaneously to pelvic nodes with a dose of $25 \mathrm{~Gy}$. Nine patients experienced grade $(G) \geq 2$ gastrointestinal (GI) or genitourinary $(\mathrm{GU})$ toxicities and five men reported $\mathrm{G} \geq 2 \mathrm{GI}$ and GU toxicity at 6 months.

In the FASTR-2, a lower dose was given on prostate gland (35 Gy), no WPRT was included, and a smaller posterior planning target volume (PTV) (4 $\mathrm{mm}$ vs $5 \mathrm{~mm}$ ) margin was provided. As expected, the FASTR-2 showed lower grades of GI/GU toxicities with respect to the FASTR trial. One patient reported a G2 GI acute toxicity, and no cases of G2 GI late toxicities were counted. Nine and five patients reported acute and late G2 GU toxicities, respectively.

From the reported results, ultra-hypofractionated RT schemes on prostate gland seems a feasible, safe, and effective treatment options for HR PCa patients.

\section{New Potential Drugs}

In order to provide novel and personalized treatment strategies and to improve QoL and long-term outcomes, drugs currently administered in metastatic settings are in study for localized and locally advanced HR PCa patients. In particular, based on the striking results in advanced $\mathrm{PCa}(44,45)$, both second-generation ADT and poly (adenosine diphosphate-ribose) polymerase 1 inhibitors (iPARP) are currently considered as potential candidates to be administered in early stages of HR PCa in order to improve oncological outcomes in this controversial setting.

\section{Second-Generation ADT}

First-generation antiandrogens established androgen receptor (AR) blockade as a therapeutic strategy but do not completely abrogate its activity (46). Despite the immediate palliative effect achieved with ADT, patients tend to relapse within a few years due to alternative mechanisms of AR signaling, AR amplification/ alternative splicing, intratumoral androgen production, or adrenal gland testosterone production.

Nowadays, efficacy and potency have been improved by the development of second-generation antiandrogen therapies which exhibits (i) increased specificity to the AR over other steroidal receptors, (ii) higher affinity than the first generation, (iii) exclusively antagonistic activity towards the $\mathrm{AR}$, and in turn, (iv) no androgen withdrawal syndrome. These secondgenerations molecules include androgen biosynthesis inhibitor abiraterone acetate and direct AR blockers, such as enzalutamide, apalutamide, and darolutamide, blocking AR with 6-9-fold greater affinity than the first-generation agents (47, 48). Hypothetically, the association of second-generation androgen receptor pathway inhibitors with EBRT can result in an added benefit for patients, especially those at a high risk of micrometastatic disease. From this perspective, abiraterone [STAMPEDE (49)], enzalutamide (ENZARAD), and apalutamide (ATLAS (50), ARNEO (51), PROTEUS) are currently under investigation to treat $\mathrm{HR} \mathrm{PCa}$, in combination with local approaches (Table 1).

In general, since the potential for the novel antiandrogens as standalone therapeutic had reached a plateau for use in advanced $\mathrm{PCa}$, it is far more likely that the next wave of therapeutic investigation will be focused on the combination of this class of antiandrogen therapy with other treatments such as RT and chemotherapy. In fact, as reported by Elsesy et al. (52), the use of second-generation antiandrogens radiosensitizes PCa via the inhibition of the DNA double-strand break repair machinery. These results are in accordance with recent preclinical studies (53) reporting that enzalutamide has a radiosentization role, increasing the effect of ionizing radiation.

To foster this evidence, Zhang and Colleagues (54) demonstrated that apalutamide acts as a radiosensitizer in both androgen-dependent PCa and castration-resistant PCa models. These results suggest that apalutamide can be used in combination with EBRT for the treatment of androgendependent localized PCa (50).

\section{PARP Inhibitors}

One of the potential reasons for radioresistance is the ability of tumor cells to repair the damage inflicted by radiotherapy. Following the induction of DNA double-strand breaks (DSB) by ionizing radiation, cancer cells mount a rapid response involving an extensive network of pathways. This response involves the cellular machinery required to repair damaged DNA and allows the malignant cell to survive and retain its reproductive integrity. This network is broadly referred to as the DNA damage response (DDR). It is well-known how high rates of genomic mutations in DDR genes result directly related to multiple malignancies (55-57), and more recently, it has been 
TABLE 1 | Ongoing trials.

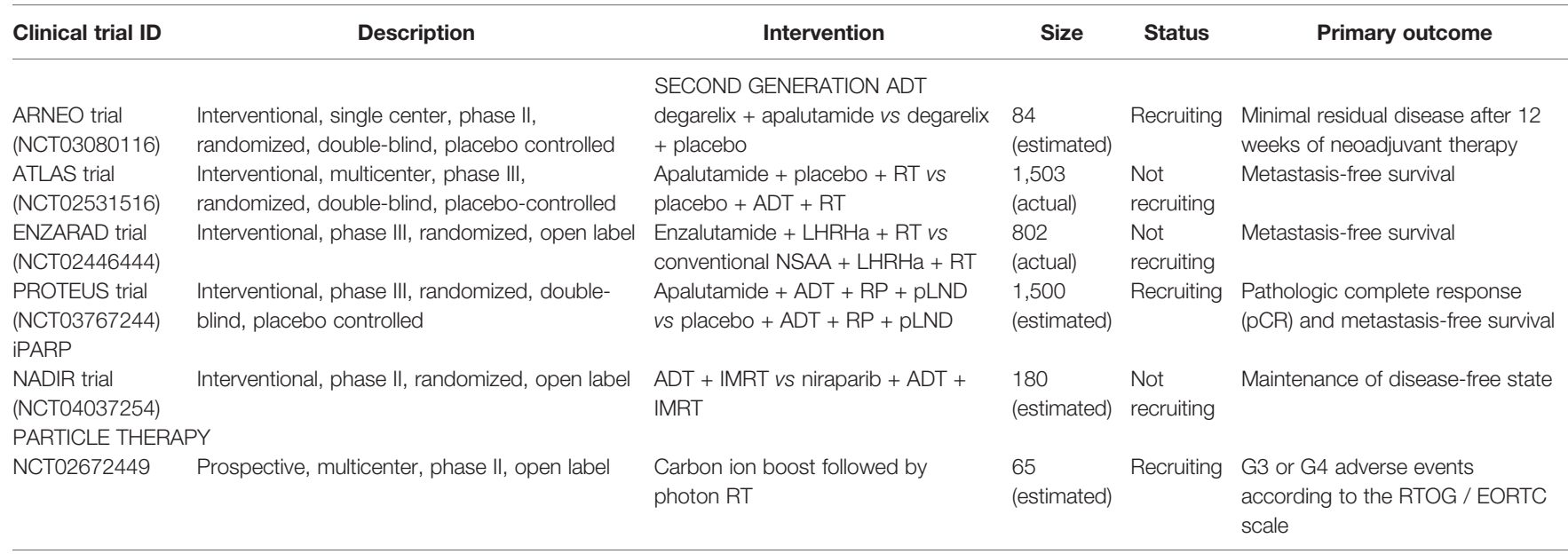

ADT, androgen deprivation therapy; G, grade; IMRT, intensity-modulated RT; LHRHa, luteinizing hormone-releasing hormone analog; NSAA, non-steroidal anti-androgen; pCR, pathologic complete response; $P L N D$, pelvic lymph node dissection; RP, radical prostatectomy; RT, radiotherapy.

suggested that tumors with such homologous recombination defects may be sensitive to $\operatorname{iPARP}(58,59)$.

Currently, there are multiple agents such as olaparib, niraparib, and rucaparib $(58,60,61)$ that target the DDR pathway. Among these iPARP, olaparib and rucaparib have been shown to be effective in men with metastatic castrationresistant PCa (mCRPC) $(44,62,63)$. Since DDR pathway alterations were seen at similar rate between localized and metastatic PCa, it has been speculated that iPARP may also have a therapeutic effect in localized PCa (64).

To support this hypothesis, a 2019 study by Kim et al. (64) analyzed the DDR pathway alterations in localized PCa using The Cancer Genome Atlas (TCGA) public database. Their results highlighted that DDR alteration rate was surprisingly higher than suggested by previous studies $(65,66)$ and was associated with shorter OS in men with postoperatively HR features.

Some of the ongoing trials regrading iPARPs in localized PCa are reported in Table $\mathbf{1}$.

Overall, the above reported findings suggest that a dysregulated DDR pathway may occur earlier during PCa progression than previously thought and that available inhibitors of DDR pathway, such as iPARPs, may have an effective therapeutic role in localized PCa.

\section{Particle Therapy}

Particle therapy has been gaining growing interest due to the particular physical and radiobiological properties of protons and other heavy ions, including carbon ions, compared to photons (67). Particularly, hadrontherapy with protons and carbon ions has been considered a suitable strategy for the treatment of localized and locally advanced PCa to reach high doses while maintaining a lower toxicity rate.

\section{Carbon Ion Therapy}

Carbon ion RT (CIRT) may represent an ideal treatment method for PCa due to the unique physical and biological advantages of carbon ion beams. The dose distribution of CIRT is most advantageous for EBRT techniques because of its superior dose characteristics (68). Firstly, steep dose gradients result in a better sparing of organs at risk (OARs) close to the target. Moreover, carbon ion beams have a high relative biological effectiveness (RBE), resulting from a high linear energy transfer, with their effect estimated to be approximately three times those of photons and protons $(69,70)$. Finally, carbon ions might affect radioresistant clusters and make them more sensitive to a subsequent photon therapy.

The first clinical trial of CIRT for PCa was initiated at the National Institute of Radiological Sciences (NIRS) in 1994, and the efficacy and feasibility of CIRT for localized PCa have been demonstrated through three phase I/II and two phase II clinical trials (71) at NIRS. The studies published from the Japanese centers represent an important starting point for the clinical use of carbon ions in this setting of patients $(72,73)$.

A 2017 study by Kasuya and Colleagues (74) analyzed the treatment outcomes of HR localized PCa treated with CIRT + ADT compared with standard treatment modalities, focusing on PCa-specific mortality (PCSM). Despite differences in PCSM among the high-risk groups, CIRT combined with ADT yielded relatively favorable treatment outcomes.

The first prospective observational study conducted at a facility other than NIRS is the study by Kawamura et al. (75), which reported low GU and GI toxicities with good biochemical control within 5 years following moderately hypofractionated CIRT for localized PCa.

The NCT02672449 is a prospective, multicentric, phase II openlabel trial that might provide novel insights on a new mixed beam RT scheme of a carbon ion boost followed by pelvic photon RT (76), and details about this ongoing trial are reported in Table 1. Overall, data about CIRT in HR setting seems encouraging and could provide novel insight for the treatment of these patients.

\section{Proton Therapy}

Owing to the well-known unique dose distribution of protons (77), efforts have been made to adapt their benefits in PCa therapies. In particular, their ability to reduce irradiation to the 
adjacent OARs, thanks to the Bragg Peak (78-80), allows for a highly localized deposition of energy on the tumor (81).

As of today, two studies report data about PBRT in an HR setting.

Takagi et al. (82) reported the largest PBRT $( \pm$ ADT) series in localized PCa with a 10-year follow-up. Among a cohort of 2,021 patients, a total of 792 belonged to HR or very HR groups. The control of PBRT resulted favorable, with a biochemical control rate of 68 and $62 \%$ in HR and very HR patients, respectively. Five-year OS was $96 \%$ in the HR group and $92 \%$ in the very HR cohort. The results of the study encourage the planning of novel prospective clinical trials.

In a smallest series, Arimura et al. (83) conducted a prospective cohort study on 218 patients with intermediate-risk and HR PCa declining ADT, receiving PBRT. Unexpectedly, results were similar to those of previous reported ones from studies concerning PBRT + ADT where in a PBRT setting, ADT for 12 months and 21 months was shown as preferable for HR PCa patients (84). Therefore, monotherapy PBRT can be considered as an optional treatment in this setting, even if studies that include more patients and longer follow-up are needed to clarify the definitive role of PBRT in the treatment of HR localized PCa.

In particular, there is an urgent need for randomized data comparing photon- versus proton-based EBRT head to-head for localized and HR PCa cancer to rigorously inform the debate surrounding proton therapy for PCa.

\section{CONCLUSIONS}

Some of the old questions in the treatment of HR localized PCa seem to have been solved; nevertheless, modern treatment strategies bring with them novel questions that need to be addressed.

Multidisciplinary teams of urologists, medical oncologists, radiation oncologists, radiologists, and pathologists will be instrumental in shifting the treatment tide for the patients.

Integrative multimodal personalized treatment approaches inclusive of surgery, ultra-hypofractionated RT, hadrontherapy, and systemic therapy represent a valid potential way to improve long-term outcomes in patients with HR PCa.

\section{AUTHOR CONTRIBUTIONS}

GMa, GC, and MZ were responsible for conception and design of the study and wrote the first draft of the manuscript. SV and MP were responsible for data acquisition and wrote sections of the manuscript. MA wrote sections of the manuscript. All authors contributed to the article and approved the submitted version.

\section{FUNDING}

Università degli Studi di Milano. This study was supported by a research grant from the Associazione Italiana per la Ricerca sul Cancro (AIRC) entitled“ Radioablation \pm hormonotherapy for prostate cancer oligorecurrences (RADIOSA trial): potential of imaging and biology" registered at ClinicalTrials.gov NCT03940235, approved by the Ethics Committee of IRCCS Istituto Europeo di Oncologia and Centro Cardiologico Monzino (IEO-997).

\section{REFERENCES}

1. Mottet N, van den Bergh RCN, Briers E, Bourke L, Cornford P, De Santis M, et al. EAU-ESUR-ESTRO-SIOG-Guidelines-On-Prostate-Cancer.

2. Sundi D, Tosoian JJ, Nyame YA, Alam R, Achim M, Reichard CA, et al. Outcomes of Very High-Risk Prostate Cancer After Radical Prostatectomy: Validation Study From 3 Centers. Cancer (2019) 125:391-7. doi: 10.1002/ cncr.31833

3. Zagars GK, Pollack A, von Eschenbach AC. Prognostic Factors for Clinically Localized Prostate Carcinoma: Analysis of 938 Patients Irradiated in the Prostate Specific Antigen Era. Cancer (1997) 79:1370-80. doi: 10.1002/(SICI) 1097-0142(19970401)79:7<1370::AID-CNCR15>3.0.CO;2-X

4. Jackson W, Hamstra DA, Johnson S, Zhou J, Foster B, Foster C, et al. Gleason Pattern 5 is the Strongest Pathologic Predictor of Recurrence, Metastasis, and Prostate Cancer-Specific Death in Patients Receiving Salvage Radiation Therapy Following Radical Prostatectomy. Cancer (2013) 119:3287-94. doi: $10.1002 /$ cncr.28215

5. Mossanen M, Nepple KG, Grubb RL 3rd, Androile GL, Kallogjeri D, Klein EA, et al. Heterogeneity in Definitions of High-Risk Prostate Cancer and Varying Impact on Mortality Rates After Radical Prostatectomy. Eur Urol Oncol (2018) 1:143-8. doi: 10.1016/j.euo.2018.02.004

6. Knipper S, Karakiewicz PI, Heinze A, Preisser F, Steuber T, Huland H, et al. Definition of High-Risk Prostate Cancer Impacts Oncological Outcomes After Radical Prostatectomy. Urol Oncol (2020) 38:184-90. doi: 10.1016/ j.urolonc.2019.12.014

7. Frandsen J, Orton A, Shrieve D, Tward J. Risk of Death From Prostate Cancer With and Without Definitive Local Therapy When Gleason Pattern 5 Is

Present: A Surveillance, Epidemiology, and End Results Analysis. Cureus (2017) 9:e1453. doi: 10.7759/cureus.1453

8. Schaeffer E, Srinivas S, Antonarakis SE, Armstrong AJ, Bekelman JE, Cheng $\mathrm{H}$, et al. National Comprehensive Cancer Network (NCCN) Prostate Cancer Guidelines 2021.

9. Moris L, Cumberbatch MG, Van den Broeck T, Gandaglia G, Fossati N, Kelly B, et al. Benefits and Risks of Primary Treatments for High-Risk Localized and Locally Advanced Prostate Cancer: An International Multidisciplinary Systematic Review. Eur Urol (2020) 77:614-27. doi: 10.1016/ j.eururo.2020.01.033

10. Stranne J, Brasso K, Brennhovd B, Johansson E, Jäderling F, Kouri M, et al. SPCG-15: A Prospective Randomized Study Comparing Primary Radical Prostatectomy and Primary Radiotherapy Plus Androgen Deprivation Therapy for Locally Advanced Prostate Cancer. Scand J Urol (2018) 52:31320. doi: 10.1080/21681805.2018.1520295

11. Warde P, Mason M, Ding K, Kirkbride P, Brundage M, Cowan R, et al. Combined Androgen Deprivation Therapy and Radiation Therapy for Locally Advanced Prostate Cancer: A Randomised, Phase 3 Trial. Lancet Lond Engl (2011) 378:2104-11. doi: 10.1016/S0140-6736(11)61095-7

12. Bolla M, Van Tienhoven G, Warde P, Dubois JB, Mirimanoff RO, Storme G, et al. External Irradiation With or Without Long-Term Androgen Suppression for Prostate Cancer With High Metastatic Risk: 10-Year Results of an EORTC Randomised Study. Lancet Oncol (2010) 11:1066-73. doi: 10.1016/S1470-2045(10)70223-0

13. Widmark A, Klepp O, Solberg A, Damber JE, Angelsen A, Fransson P, et al. Endocrine Treatment, With or Without Radiotherapy, in Locally Advanced Prostate Cancer (SPCG-7/SFUO-3): An Open Randomised Phase III Trial. Lancet Lond Engl (2009) 373:301-8. doi: 10.1016/S0140-6736(08)61815-2 
14. Lawton CAF, Lin X, Hanks GE, Lepor H, Grignon DJ, Brereton HD, et al. Duration of Androgen Deprivation in Locally Advanced Prostate Cancer: Long-Term Update of NRG Oncology RTOG 9202. Int J Radiat Oncol Biol Phys (2017) 98:296-303. doi: 10.1016/j.ijrobp.2017.02.004

15. Bolla M, de Reijke TM, Van Tienhoven G, Van den Bergh AC, Oddens J, Poortmans PM, et al. Duration of Androgen Suppression in the Treatment of Prostate Cancer. N Engl J Med (2009) 360:2516-27. doi: 10.1056/ NEJMoa0810095

16. Nabid A, Carrier N, Martin AG, Bahary JP, Lemaire C, Vass S, et al. Duration of Androgen Deprivation Therapy in High-Risk Prostate Cancer: A Randomized Phase III Trial. Eur Urol (2018) 74:432-41. doi: 10.1016/ j.eururo.2018.06.018

17. Roach M 3rd, Bae K, Speight J, Wolkov HB, Rubin P, Lee RJ, et al. Short-Term Neoadjuvant Androgen Deprivation Therapy and External-Beam Radiotherapy for Locally Advanced Prostate Cancer: Long-Term Results of RTOG 8610. J Clin Oncol Off J Am Soc Clin Oncol (2008) 26:585-91. doi: 10.1200/JCO.2007.13.9881

18. Denham JW, Steigler A, Lamb DS, Joseph D, Turner S, Matthews J, et al. Short-Term Neoadjuvant Androgen Deprivation and Radiotherapy for Locally Advanced Prostate Cancer: 10-Year Data From the TROG 96.01 Randomised Trial. Lancet Oncol (2011) 12:451-9. doi: 10.1016/S1470-2045 (11)70063-8

19. D’Amico AV, Chen M-H, Renshaw AA, Loffredo M, Kantoff PW. Androgen Suppression and Radiation vs Radiation Alone for Prostate Cancer: A Randomized Trial. JAMA (2008) 299:289-95. doi: 10.1001/ jama.299.3.289

20. Roach M, Moughan J, Lawton CAF, Dicker AP, Zeitzer KL, Gore EM, et al. Sequence of Hormonal Therapy and Radiotherapy Field Size in Unfavourable, Localised Prostate Cancer (NRG/RTOG 9413): Long-Term Results of a Randomised, Phase 3 Trial. Lancet Oncol (2018) 19:1504-15. doi: 10.1016/ S1470-2045(18)30528-X

21. Lawton CA, DeSilvio M, Roach M 3rd, Uhl V, Kirsch R, Seider M, et al. An Update of the Phase III Trial Comparing Whole Pelvic to Prostate Only Radiotherapy and Neoadjuvant to Adjuvant Total Androgen Suppression: Updated Analysis of RTOG 94-13, With Emphasis on Unexpected Hormone/ Radiation Interactions. Int J Radiat Oncol Biol Phys (2007) 69:646-55. doi: 10.1016/j.ijrobp.2007.04.003

22. Murthy V, Maitre P, Kannan S, Panigrahi G, Krishnatry R, Bakshi G, et al. Prostate-Only Versus Whole-Pelvic Radiation Therapy in High-Risk and Very High-Risk Prostate Cancer (POP-RT): Outcomes From Phase III Randomized Controlled Trial. J Clin Oncol Off J Am Soc Clin Oncol (2021) 39:1234-42. doi: 10.1200/JCO.20.03282

23. Spratt DE, Vargas HA, Zumsteg ZS, Golia Pernicka JS, Osborne JR, Pei X, et al. Patterns of Lymph Node Failure After Dose-Escalated Radiotherapy: Implications for Extended Pelvic Lymph Node Coverage. Eur Urol (2017) 71:37-43. doi: 10.1016/j.eururo.2016.07.043

24. Syndikus I, Cruickshank C, Staffurth J, Tree A, Henry A, Naismith O, et al. PIVOTALboost: A Phase III Randomised Controlled Trial of Prostate and Pelvis Versus Prostate Alone Radiotherapy With or Without Prostate Boost (CRUK/16/018). Clin Transl Radiat Oncol (2020) 25:22-8. doi: 10.1016/ j.ctro.2020.08.003

25. Ong WL, Koh TL, Lim Joon D, Chao M, Farrugia B, Lau E, et al. ProstateSpecific Membrane Antigen-Positron Emission Tomography/Computed Tomography (PSMA-PET/CT)-Guided Stereotactic Ablative Body Radiotherapy for Oligometastatic Prostate Cancer: A Single-Institution Experience and Review of the Published Literature. BJU Int (2019) 124 (Suppl 1):19-30. doi: 10.1111/bju.14886

26. Perera M, Papa N, Roberts M, Williams M, Udovicich C, Vela I, et al. Gallium68 Prostate-Specific Membrane Antigen Positron Emission Tomography in Advanced Prostate Cancer-Updated Diagnostic Utility, Sensitivity, Specificity, and Distribution of Prostate-Specific Membrane Antigen-Avid Lesions: A Systematic Review and Meta-Analysis. Eur Urol (2020) 77:403-17. doi: 10.1016/j.eururo.2019.01.049

27. Tharmalingam H, Choudhury A, Van Herk M, McWilliam A, Hoskin PJ. New Approaches for Effective and Safe Pelvic Radiotherapy in High-Risk Prostate Cancer. Nat Rev Urol (2019) 16:523-38. doi: 10.1038/s41585-019-0213-3

28. Catton CN, Lukka H, Gu CS, Martin JM, Supiot S, Chung PWM, et al. Randomized Trial of a Hypofractionated Radiation Regimen for the
Treatment of Localized Prostate Cancer. J Clin Oncol Off J Am Soc Clin Oncol (2017) 35:1884-90. doi: 10.1200/JCO.2016.71.7397

29. Dearnaley D, Syndikus I, Mossop H, Khoo V, Birtle A, Bloomfield D, et al. Conventional Versus Hypofractionated High-Dose Intensity-Modulated Radiotherapy for Prostate Cancer: 5-Year Outcomes of the Randomised, non-Inferiority, Phase 3 CHHiP Trial. Lancet Oncol (2016) 17:1047-60. doi: 10.1016/S1470-2045(16)30102-4

30. Incrocci L, Wortel RC, Alemayehu WG, Aluwini S, Schimmel E, Krol S. Hypofractionated Versus Conventionally Fractionated Radiotherapy for Patients With Localised Prostate Cancer (HYPRO): Final Efficacy Results From a Randomised, Multicentre, Open-Label, Phase 3 Trial. Lancet Oncol (2016) 17:1061-9. doi: 10.1016/S1470-2045(16)30070-5

31. Lee WR, Dignam JJ, Amin MB, Bruner DW, Low D, Swanson GP, et al. Randomized Phase III Noninferiority Study Comparing Two Radiotherapy Fractionation Schedules in Patients With Low-Risk Prostate Cancer. J Clin Oncol Off J Am Soc Clin Oncol (2016) 34:2325-32. doi: 10.1200/ JCO.2016.67.0448

32. Gulliford S, Hall E, Dearnaley D. Hypofractionation Trials and Radiobiology of Prostate Cancer. Oncoscience (2017) 4(3-4):27-28. doi: 10.18632/ oncoscience. 347

33. Miralbell R, Roberts SA, Zubizarreta E, Hendry JH. Dose-Fractionation Sensitivity of Prostate Cancer Deduced From Radiotherapy Outcomes of 5,969 Patients in Seven International Institutional Datasets: $\alpha / \beta=1.4(0.9-2.2)$ Gy. Int J Radiat Oncol Biol Phys (2012) 82:e17-24. doi: 10.1016/ j.ijrobp.2010.10.075

34. Royce TJ, Lee DH, Keum N, Permpalung N, Chiew CJ, Epstein S, et al. Conventional Versus Hypofractionated Radiation Therapy for Localized Prostate Cancer: A Meta-Analysis of Randomized Noninferiority Trials. Eur Urol Focus (2019) 5:577-84. doi: 10.1016/j.euf.2017.10.011

35. Arcangeli G, Saracino B, Arcangeli S, Gomellini S, Petrongari MG, Sanguineti G, et al. Moderate Hypofractionation in High-Risk, Organ-Confined Prostate Cancer: Final Results of a Phase III Randomized Trial. J Clin Oncol Off J Am Soc Clin Oncol (2017) 35:1891-7. doi: 10.1200/JCO.2016.70.4189

36. Wilkins A, Mossop H, Syndikus I, Khoo V, Bloomfield D, Parker C, et al. Hypofractionated Radiotherapy Versus Conventionally Fractionated Radiotherapy for Patients With Intermediate-Risk Localised Prostate Cancer: 2-Year Patient-Reported Outcomes of the Randomised, nonInferiority, Phase 3 CHHiP Trial. Lancet Oncol (2015) 16:1605-16. doi: 10.1016/S1470-2045(15)00280-6

37. Al-Mamgani A, Heemsbergen WD, Peeters STH, Lebesque JV. Role of Intensity-Modulated Radiotherapy in Reducing Toxicity in Dose Escalation for Localized Prostate Cancer. Int J Radiat Oncol Biol Phys (2009) 73:685-91. doi: 10.1016/j.ijrobp.2008.04.063

38. Jani AB, Su A, Correa D, Gratzle J. Comparison of Late Gastrointestinal and Genitourinary Toxicity of Prostate Cancer Patients Undergoing IntensityModulated Versus Conventional Radiotherapy Using Localized Fields. Prostate Cancer Prostatic Dis (2007) 10:82-6. doi: 10.1038/sj.pcan.4500910

39. Morgan SC, Hoffman K, Loblaw DA, Buyyounouski MK, Patton C, Barocas D, et al. Hypofractionated Radiation Therapy for Localized Prostate Cancer: Executive Summary of an ASTRO, ASCO, and AUA Evidence-Based Guideline. Pract Radiat Oncol (2018) 8:354-60. doi: 10.1016/j.prro. 2018.08.002

40. Widmark A, Gunnlaugsson A, Beckman L, Thellenberg-Karlsson C, Hoyer M, Lagerlund M, et al. Ultra-Hypofractionated Versus Conventionally Fractionated Radiotherapy for Prostate Cancer: 5-Year Outcomes of the HYPO-RT-PC Randomised, non-Inferiority, Phase 3 Trial. Lancet Lond Engl (2019) 394:385-95. doi: 10.1016/S0140-6736(19)31131-6

41. Draulans C, van der Heide UA, Haustermans K, Pos FJ, van der Voort van Zyp J, De Boer H, et al. Primary Endpoint Analysis of the Multicentre Phase II Hypo-FLAME Trial for Intermediate and High Risk Prostate Cancer. Radiother Oncol J Eur Soc Ther Radiol Oncol (2020) 147:92-8. doi: 10.1016/ j.radonc.2020.03.015

42. Bauman G, Ferguson M, Lock M, Chen J, Ahmad B, Venkatesan VM, et al. A Phase 1/2 Trial of Brief Androgen Suppression and Stereotactic Radiation Therapy (FASTR) for High-Risk Prostate Cancer. Int J Radiat Oncol Biol Phys (2015) 92:856-62. doi: 10.1016/j.ijrobp.2015.02.046

43. Callan L, Bauman G, Chen J, Lock M, Sexton T, D'Souza D, et al. A Phase I/II Trial of Fairly Brief Androgen Suppression and Stereotactic Radiation Therapy for 
High-Risk Prostate Cancer (FASTR-2): Preliminary Results and Toxicity Analysis. Adv Radiat Oncol (2019) 4:668-73. doi: 10.1016/j.adro.2019.07.007

44. Abida W, Patnaik A, Campbell D, Shapiro J, Bryce AH, McDermott R, et al. Rucaparib in Men With Metastatic Castration-Resistant Prostate Cancer Harboring a BRCA1 or BRCA2 Gene Alteration. J Clin Oncol Off J Am Soc Clin Oncol (2020) 38:3763-72. doi: 10.1200/JCO.20.01035

45. Fizazi K, Tran N, Fein L, Matsubara N, Rodriguez-Antolin A, Alekseev BY, et al. Abiraterone Plus Prednisone in Metastatic, Castration-Sensitive Prostate Cancer. N Engl J Med (2017) 377:352-60. doi: 10.1056/NEJMoa1704174

46. Denis LJ, Griffiths K. Endocrine Treatment in Prostate Cancer. Semin Surg Oncol (2000) 18:52-74. doi: 10.1002/(SICI)1098-2388(200001/02)18:1<52:: AID-SSU8>3.0.CO;2-6

47. Soifer HS, Souleimanian N, Wu S, Voskresenskiy AM, Collak FK, Cinar B, et al. Direct Regulation of Androgen Receptor Activity by Potent CYP17 Inhibitors in Prostate Cancer Cells. J Biol Chem (2012) 287:3777-87. doi: 10.1074/jbc.M111.261933

48. Richards J, Lim AC, Hay CW, Taylor AE, Wingate A, Nowakowska K, et al. Interactions of Abiraterone, Eplerenone, and Prednisolone With Wild-Type and Mutant Androgen Receptor: A Rationale for Increasing Abiraterone Exposure or Combining With MDV3100. Cancer Res (2012) 72:2176-82. doi: 10.1158/0008-5472.CAN-11-3980

49. James ND, de Bono JS, Spears MR, Clarke NW, Mason MD, Dearnaley DP, et al. Abiraterone for Prostate Cancer Not Previously Treated With Hormone Therapy. N Engl J Med (2017) 377:338-51. doi: 10.1056/NEJMoa1702900

50. Sandler HM, McKenzie MR, Tombal BF, Baskin-Bey E, Freedland SJ, Roach M, et al. ATLAS: A Randomized, Double-Blind, Placebo-Controlled, Phase 3 Trial of Apalutamide (ARN-509) in Patients With High-Risk Localized or Locally Advanced Prostate Cancer Receiving Primary Radiation Therapy. J Clin Oncol (2016) 34(15_suppl):TPS5087-TPS5087. doi: 10.1200/ JCO.2016.34.15_suppl.TPS5087

51. Tosco L, Laenen A, Gevaert T, Salmon I, Decaestecker C, Davicioni E, et al. Neoadjuvant Degarelix With or Without Apalutamide Followed by Radical Prostatectomy for Intermediate and High-Risk Prostate Cancer: ARNEO, a Randomized, Double Blind, Placebo-Controlled Trial. BMC Cancer (2018) 18:354. doi: 10.1186/s12885-018-4275-z

52. Elsesy ME, Oh-Hohenhorst SJ, Löser A, Oing C, Mutiara S, Köcher S, et al. Second-Generation Antiandrogen Therapy Radiosensitizes Prostate Cancer Regardless of Castration State Through Inhibition of DNA Double Strand Break Repair. Cancers (2020) 12(9):2467. doi: 10.3390/cancers12092467

53. Ghashghaei M, Niazi TM, Heravi M, Bekerat H, Trifiro M, Paliouras M, et al. Enhanced Radiosensitization of Enzalutamide via Schedule Dependent Administration to Androgen-Sensitive Prostate Cancer Cells. Prostate (2018) 78:64-75. doi: 10.1002/pros.23445

54. Zhang W, Liao CY, Chtatou H, Incrocci L, van Gent DC, van Weerden WM, et al. Apalutamide Sensitizes Prostate Cancer to Ionizing Radiation via Inhibition of Non-Homologous End-Joining DNA Repair. Cancers (2019) 11(10):1593. doi: 10.2139/ssrn.3454677

55. van Gent DC, Kanaar R. Exploiting DNA Repair Defects for Novel Cancer Therapies. Mol Biol Cell (2016) 27:2145-8. doi: 10.1091/mbc.E15-10-0698

56. Oda K, Tanikawa M, Sone K, Mori-Uchino M, Osuga Y, Fujii T. Recent Advances in Targeting DNA Repair Pathways for the Treatment of Ovarian Cancer and Their Clinical Relevance. Int J Clin Oncol (2017) 22:611-8. doi: 10.1007/s10147-017-1137-7

57. O'Connor MJ. Targeting the DNA Damage Response in Cancer. Mol Cell (2015) 60:547-60. doi: 10.1016/j.molcel.2015.10.040

58. Lorusso D, Tripodi E, Maltese G, Lepori S, Sabatucci I, Bogani G, et al. Spotlight on Olaparib in the Treatment of BRCA-Mutated Ovarian Cancer: Design, Development and Place in Therapy. Drug Des Devel Ther (2018) 12:1501-9. doi: 10.2147/DDDT.S124447

59. Gavande NS, VanderVere-Carozza PS, Hinshaw HD, Jalal SI, Sears CR, Pawelczak KS, et al. DNA Repair Targeted Therapy: The Past or Future of Cancer Treatment? Pharmacol Ther (2016) 160:65-83. doi: 10.1016/j.pharmthera.2016.02.003

60. Berek JS, Matulonis UA, Peen U, Ghatage P, Mahner S, Redondo A, et al. Safety and Dose Modification for Patients Receiving Niraparib. Ann Oncol Off J Eur Soc Med Oncol (2018) 29:1784-92. doi: 10.1093/annonc/mdy181

61. Dal Molin GZ, Omatsu K, Sood AK, Coleman RL. Rucaparib in Ovarian Cancer: An Update on Safety, Efficacy and Place in Therapy. Ther Adv Med Oncol (2018) 10:1758835918778483. doi: 10.1177/1758835918778483
62. Mateo J, Carreira S, Sandhu S, Miranda S, Mossop H, Perez-Lopez R, et al. DNA-Repair Defects and Olaparib in Metastatic Prostate Cancer. N Engl J Med (2015) 373:1697-708. doi: 10.1056/NEJMoa1506859

63. Clarke N, Wiechno P, Alekseev B, Sala N, Jones R, Kocak I, et al. Olaparib Combined With Abiraterone in Patients With Metastatic Castration-Resistant Prostate Cancer: A Randomised, Double-Blind, Placebo-Controlled, Phase 2 Trial. Lancet Oncol (2018) 19:975-86. doi: 10.1016/S1470-2045(18)30365-6

64. Kim IE Jr, Kim S, Srivastava A, Saraiya B, Mayer T, Kim WJ, et al. Similar Incidence of DNA Damage Response Pathway Alterations Between Clinically Localized and Metastatic Prostate Cancer. BMC Urol (2019) 19:33. doi: 10.1186/s12894-019-0453-9

65. Cancer Genome Atlas Research Network. The Molecular Taxonomy of Primary Prostate Cancer. Cell (2015) 163:1011-25. doi: 10.1016/j.cell.2015.10.025

66. Marshall CH, Fu W, Wang H, Baras AS, Lotan TL, Antonarakis ES, et al. Prevalence of DNA Repair Gene Mutations in Localized Prostate Cancer According to Clinical and Pathologic Features: Association of Gleason Score and Tumor Stage. Prostate Cancer Prostatic Dis (2019) 22:59-65. doi: 10.1038/ s41391-018-0086-1

67. Malouff TD, Mahajan A, Krishnan S, Beltran C, Seneviratne DS, Trifiletti DM. Carbon Ion Therapy: A Modern Review of an Emerging Technology. Front Oncol (2020) 10:82. doi: 10.3389/fonc.2020.00082

68. Georg D, Hopfgartner J, Gòra J, Kuess P, Kragl G, Berger D, et al. Dosimetric Considerations to Determine the Optimal Technique for Localized Prostate Cancer Among External Photon, Proton, or Carbon-Ion Therapy and HighDose-Rate or Low-Dose-Rate Brachytherapy. Int J Radiat Oncol Biol Phys (2014) 88:715-22. doi: 10.1016/j.ijrobp.2013.11.241

69. Held KD, Kawamura H, Kaminuma T, Paz AE, Yoshida Y, Liu Q, et al. Effects of Charged Particles on Human Tumor Cells. Front Oncol (2016) 6:23. doi: 10.3389/fonc.2016.00023

70. Durante M, Orecchia R, Loeffler IS. Charged-Particle Therapy in Cancer: Clinical Uses and Future Perspectives. Nat Rev Clin Oncol (2017) 14:483-95. doi: 10.1038/nrclinonc.2017.30

71. Ishikawa H, Tsuji H, Kamada T, Akakura K, Suzuki H, Shimazaki J, et al. Carbon-Ion Radiation Therapy for Prostate Cancer. Int I Urol Off J Jpn Urol Assoc (2012) 19:296-305. doi: 10.1111/j.1442-2042.2012.02961.x

72. Nomiya T, Tsuji H, Maruyama K, Toyama S, Suzuki H, Akakura K, et al. Phase I/II Trial of Definitive Carbon Ion Radiotherapy for Prostate Cancer: Evaluation of Shortening of Treatment Period to 3 Weeks. Br J Cancer (2014) 110:2389-95. doi: 10.1038/bjc.2014.191

73. Akakura K, Tsujii H, Morita S, Tsuji H, Yagishita T, Isaka S, et al. Phase I/II Clinical Trials of Carbon Ion Therapy for Prostate Cancer. Prostate (2004) 58:252-8. doi: 10.1002/pros.10328

74. Kasuya G, Ishikawa H, Tsuji H, Haruyama Y, Kobashi G, Ebner DK, et al. Cancer-Specific Mortality of High-Risk Prostate Cancer After Carbon-Ion Radiotherapy Plus Long-Term Androgen Deprivation Therapy. Cancer Sci (2017) 108:2422-9. doi: 10.1111/cas.13402

75. Kawamura H, Kubo N, Sato H, Mizukami T, Katoh H, Ishikawa H, et al. Moderately Hypofractionated Carbon Ion Radiotherapy for Prostate Cancer; a Prospective Observational Study 'Gunma0702'. BMC Cancer (2020) 20:75. doi: 10.1186/s12885-020-6570-8

76. Marvaso G, Jereczek-Fossa BA, Vischioni B, Ciardo D, Giandini T, Hasegawa A, et al. Phase II Multi-Institutional Clinical Trial on a New Mixed Beam RT Scheme of IMRT on Pelvis Combined With a Carbon Ion Boost for High-Risk Prostate Cancer Patients. Tumori (2017) 103:314-8. doi: 10.5301/tj.5000587

77. Boyer AL, Goitein M, Lomax AJ, Pedroni ES. Radiation in the Treatment of Cancer. Phys Today (2002) 55:34-6. doi: 10.1063/1.1522213

78. Lomax AJ. Charged Particle Therapy: The Physics of Interaction. Cancer Sudbury Mass (2009) 15:285-91. doi: 10.1097/PPO.0b013e3181af5cc7

79. Brown A, Suit H. The Centenary of the Discovery of the Bragg Peak. Radiother Oncol J Eur Soc Ther Radiol Oncol (2004) 73:265-8. doi: 10.1016/ j.radonc.2004.09.008

80. Schulz-Ertner D. The Clinical Experience With Particle Therapy in Adults. Cancer J Sudbury Mass (2009) 15:306-11. doi: 10.1097/PPO.0b013e3181b01922

81. Greco C. Particle Therapy in Prostate Cancer: A Review. Prostate Cancer Prostatic Dis (2007) 10:323-30. doi: 10.1038/sj.pcan.4500987

82. Takagi M, Demizu Y, Fujii O, Terashima K, Niwa Y, Daimon T, et al. Proton Therapy for Localized Prostate Cancer: Long-Term Results From a SingleCenter Experience. Int J Radiat Oncol Biol Phys (2021) 109:964-74. doi: 10.1016/j.ijrobp.2020.11.007 
83. Arimura T, Yoshiura T, Matsukawa K, Kondo N, Kitano I, Ogino T. Proton Beam Therapy Alone for Intermediate- or High-Risk Prostate Cancer: An Institutional Prospective Cohort Study. Cancers (2018) 10(4):116. doi: $10.3390 /$ cancers 10040116

84. Murakami M, Ishikawa H, Shimizu S, Iwata H, Okimoto T, Takagi M, et al. Optimal Androgen Deprivation Therapy Combined With Proton Beam Therapy for Prostate Cancer: Results From a Multi-Institutional Study of the Japanese Radiation Oncology Study Group. Cancers (2020) 12(6):1690. doi: $10.3390 /$ cancers 12061690

Conflict of Interest: The authors declare that the research was conducted in the absence of any commercial or financial relationships that could be construed as a potential conflict of interest.

The handling editor declared a past co-authorship with one of the authors BJ.
Publisher's Note: All claims expressed in this article are solely those of the authors and do not necessarily represent those of their affiliated organizations, or those of the publisher, the editors and the reviewers. Any product that may be evaluated in this article, or claim that may be made by its manufacturer, is not guaranteed or endorsed by the publisher.

Copyright (c) 2021 Marvaso, Corrao, Zaffaroni, Pepa, Augugliaro, Volpe, Musi, Luzzago, Mistretta, Verri, Cossu Rocca, Ferro, Petralia, Nolè, De Cobelli, Orecchia and Jereczek-Fossa. This is an open-access article distributed under the terms of the Creative Commons Attribution License (CC BY). The use, distribution or reproduction in other forums is permitted, provided the original author $(s)$ and the copyright owner(s) are credited and that the original publication in this journal is cited, in accordance with accepted academic practice. No use, distribution or reproduction is permitted which does not comply with these terms. 